http://jmscr.igmpublication.org/home/ ISSN (e)-2347-176x ISSN (p) 2455-0450 crossref DOI: https://dx.doi.org/10.18535/jmscr/v9i5.07

\title{
Progressive Symmetric Erythrokeratoderma- A Rare Genodermatosis
}

\author{
Authors \\ Dr Ritu Rani ${ }^{1}$, Dr Mudita Gupta ${ }^{2 *}$, Dr Ghanshyam Verma ${ }^{3}$, Dr Reena K Sharma ${ }^{4}$ \\ ${ }^{1}$ Junior Resident, Department of Dermatology, IGMC shimla \\ ${ }^{2}$ Assistant Professor, Department of Dermatology, IGMC, Shimla \\ ${ }^{3}$ Associate Professor, Department of Dermatology, IGMC, Shimla \\ ${ }^{4}$ Dermatologist, Civil Hospital Kangra \\ *Corresponding Author \\ Dr Mudita Gupta \\ Assistant Professor, Department of Dermatology, IGMC, Shimla, India
}

\begin{abstract}
Erythrokeratoderma are disorders of keratinization characterized by variable erythema and hyperkeratinization. They may be syndromic and non syndromic. Progressive symmetric erythrokeratodema (PSEK) and eythrokeratoderma variabilis (EKV) are the non syndromic variety of erythrokeratoderma. There are about 100 cases reported worldwide. We are describing a classical case of PSEK in a 21 year male.
\end{abstract}

\section{Introduction}

Progressive symmetric erythrokeratodema (PSEK) or Gottron's syndrome is a genetic disorder first described by Darier in 1911 and renamed by Gottron in $1921 .^{1}$ It is a very rare genodermatosis with 100 cases worldwide and amongst them fewer than 10 are from India. ${ }^{2}$ Symmetrical erythematous hyperkeratotic plaques over extremities, buttocks, head and neck are characteristic of PSEK.

\section{Case Report}

A case of 21 year old male is described who presented with history of diffuse thickening of palms and soles since 2 years of age and development of psoriasiform skin lesions since 8 years of age. He had a history of similar lesions in two siblings and maternal aunt. [Figure1]

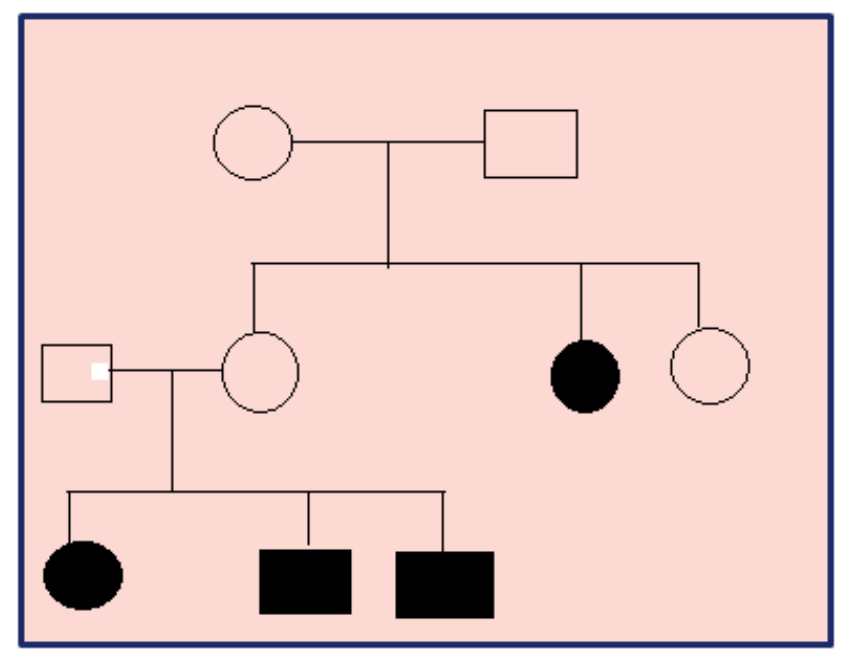

Figure 1 Showing pedigree of PSEK in the family

He was born out of non consanguineous marriage. Cutaneous lesions involved both upper and lower limbs and lumbo-sacral region. Lesions were progressive till puberty without any seasonal variation and became static afterwards. On 
examination he was a young male of average built palmo-plantar keratoderma. [Figure 2] and of normal intelligence. There was diffuse

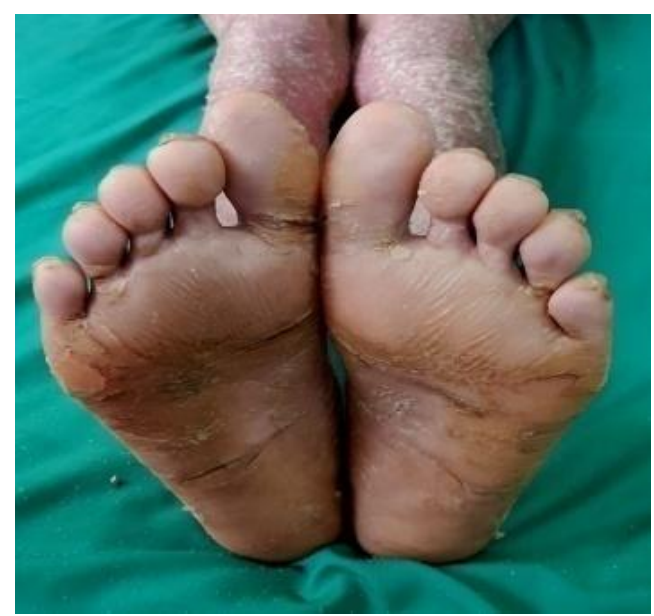

Figure 2 Showing plantar keratoderma

Multiple well defined erythematous scaly plaques were present over extensor aspect of both upper
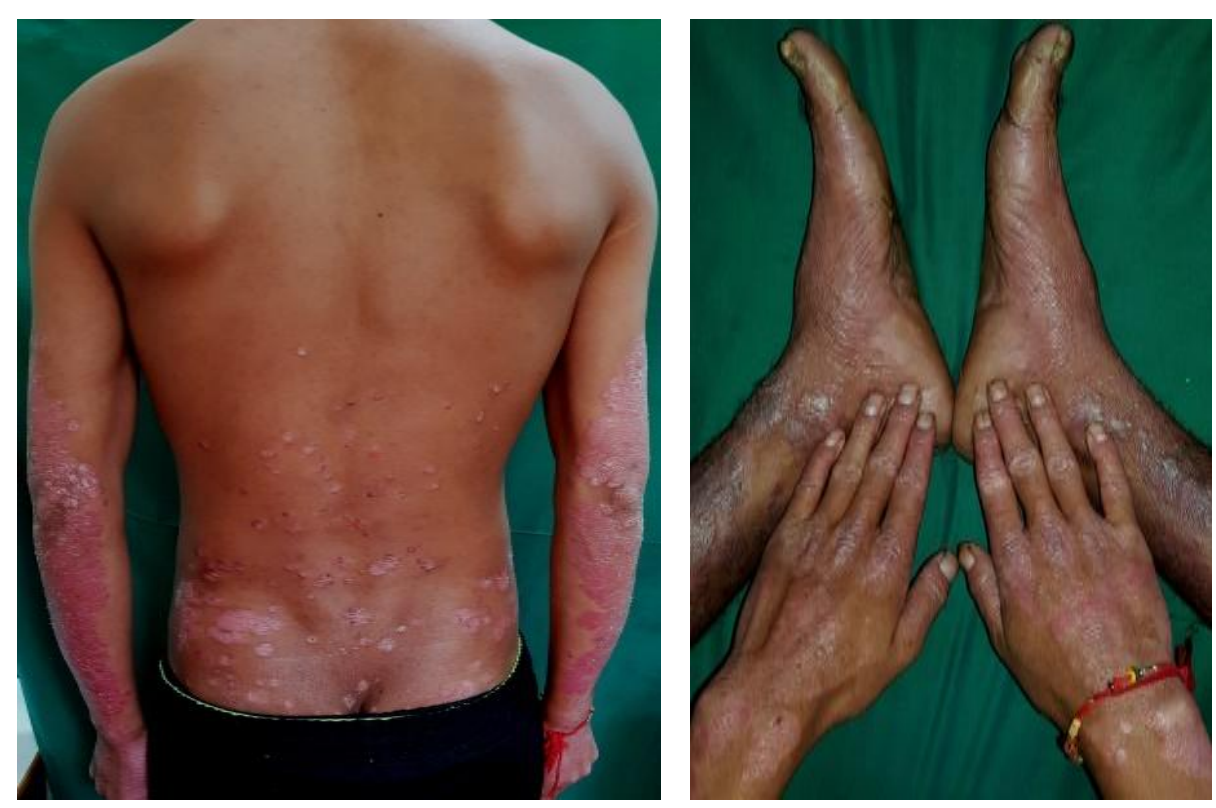

and lower limbs, lumbo-sacral region and dorsal aspect of hands and feet symmetrically [Figure 3].

Figure 3 Showing well defined erythematous, scaly symmetrical plaques on lower limbs and hands

There were no nails, teeth, mucosal or hair abnormalities. There was no other systemic involvement.

With clinical possibilities of PSEK, EKV, psoriasis and pitryasis rubra pilaris, routine investigations and biopsy were done. Demonstration of non-specific hyperkeratosis, normal granular layer, acanthosis, papillomatosis, and broad rete ridges in the upper dermis confirmed the diagnosis of PSEK. [Figure4] 


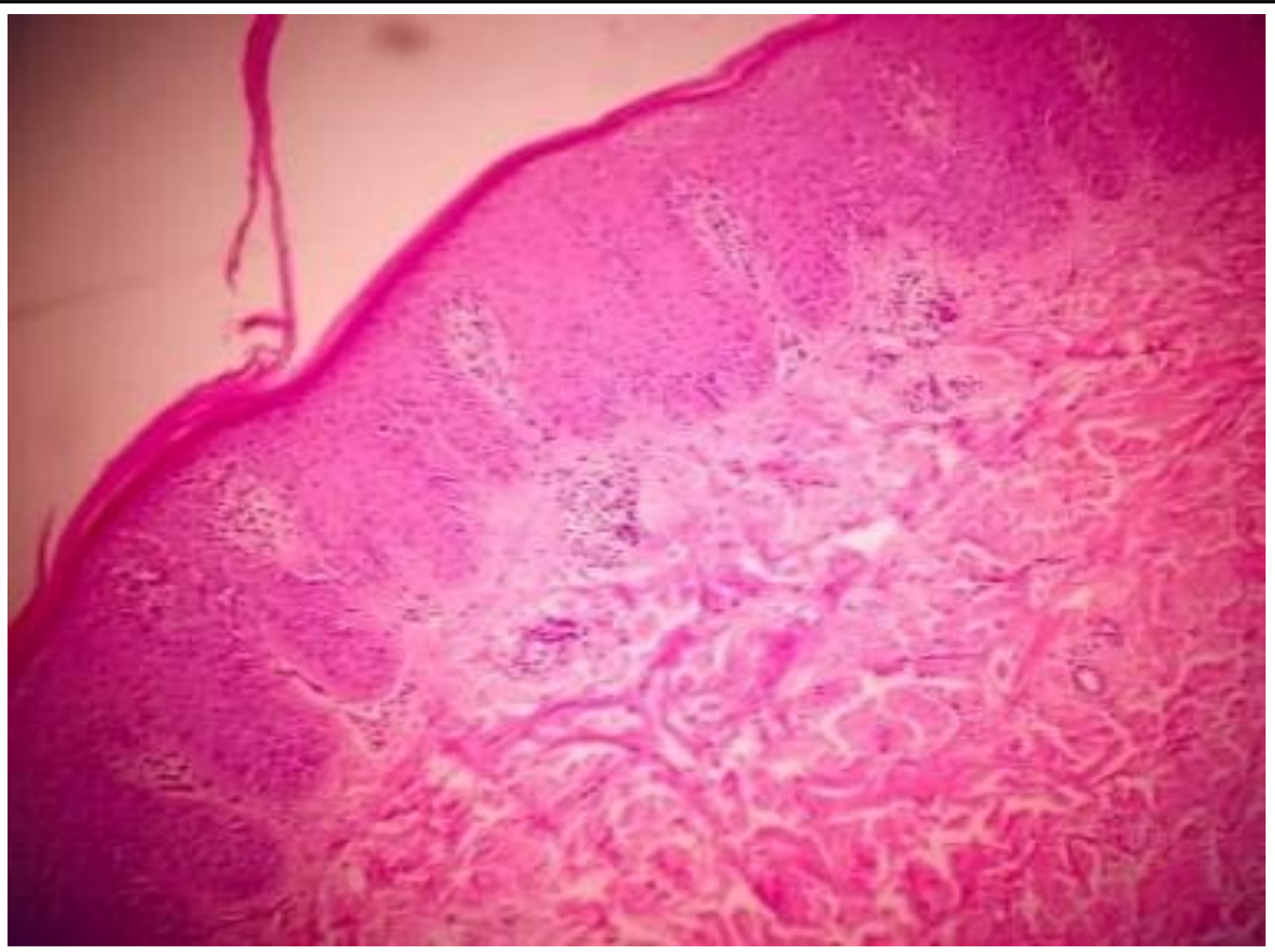

Figure 4 Histopathology (H\&E $40 \mathrm{X})$ showing hyperkeratosis acanthosis and broad rete ridges.

Genetic studies could not be done due to affordability issues. The patient was started on oral acitretin and topical emollients and advised for monthly follow up.

\section{Discussion}

Erythrokeratoderma as the name suggests is an umbrella term for a group of rare genetic skin disorders characterized by well-demarcated plaques of reddened, dry and thickened skin. They are classified into non syndromic and syndromic types. PSEK and EKV are the commoner non syndromic erythrokeratodermas. Other variants are extremely rare. PSEK is inherited by autosomal dominant mode predominantly and in $50 \%$ of cases family history is obtained. ${ }^{3}$ In rest of cases spontaneous mutations in loricrin or connexin gene results in phenotypic variations. ${ }^{2}$ The lesions in PSEK are well-demarcated, polycyclic, geographic shaped, erythematous, hyperkeratotic and mildly scaly plaques with striking symmetrical distribution over elbows, knees, dorsal hands and feet, buttocks and rarely face. Further PPK $(50 \%)$ and relative sparing of trunk characterize the PSEK. ${ }^{3}$ Apart from common papulo-squamous disorders it closely resembles to EKV. In PSEK lesions progress till puberty and later become static. . In EKV lesions are migratory in contrast to fixed, symmetrical and slowly progressive plaques of PSEK.

Psoriasis and pityriasis rubra pilaris can be differentiated from PSEK histopathologically. The histopathological findings in PSEK are nonspecific orthokeratotic hyperkeratosis, moderate acanthosis, a prominent granular layer, and dermal perivascular infiltration. There is no suprapapillary thinning of the epidermis or Munro's micro abscesses.

Generally a patient of PSEK has no systemic involvement. Associated neurological abnormalities have been described recently with PSEK. $^{4}$

Treatment of PSEK is often difficult and relapses frequent. Variable response has been observed with systemic and topical retinoids, topical emollients, topical corticosteroids, keratolytics ointments (urea, salicylic acid, propylene glycol, lactic acid, alpha hydroxy acid), coal tar, calcineurin inhibitors, and Vitamin D analogues. 
We emphasize that due to rarity and close resemblance to other dermatosis a meticulous workup is needed to avoid erroneous diagnosis and management.

\section{References}

1. Darier J. Progressive Symmetric Erythrokeratoderma verrucosa. Bull Soc Fr Dermatol Syphiligr 1911;2:252-64

2. Chander R, Jabeen M, Barara M, Kataria $\mathrm{D}$, PSEK with unusual associations. Indian J Dermatol 2014;59:317.

3. Mahajan VK, Khatri G,Chauhan PS, Mehta KS, Raina R, Gupta M. PSEK having overlapping features with EVK and lesional hypertrichosis: Is erythrokertoderma variabilis progressiva' more appropriate? Indian J Dermatol 2015; 60: 410-1

4. Verma SB, Uwe W. Progressive symmetric erythrokeratodermia with delayed intellectual milestones and convulsions. Indian Dermatol Online J. 2012; 3: 54-6. 